The AstrophysicAl Journal, 205:786-797, 1976 May 1

(C) 1976. The American Astronomical Society. All rights reserved. Printed in U.S.A.

\title{
DYNAMICS OF CO MOLECULAR CLOUDS IN THE GALAXY
}

\author{
Frank N. Bash and William L. Peters \\ University of Texas at Austin \\ Received 1975 July 28; revised 1975 October 6
}

\begin{abstract}
The ${ }^{12} \mathrm{C}^{16} \mathrm{O}$ spectral line at $115 \mathrm{GHz}$ has been observed at each degree of galactic longitude from $l=30^{\circ}$ through $60^{\circ}$ at $b=0^{\circ}$. The radial velocity of the positive-velocity terminus of the profile has been compared with that for the $\mathrm{H} \mathrm{I}, 21 \mathrm{~cm}$ line. Explanation of the velocity difference as a function of galactic longitude was attempted on the assumptions that the $\mathrm{CO}$ has the same kinematics as the $\mathrm{H} \mathrm{I}$, that the gas kinematics were described by (1) pure circular rotation, (2) the linear density wave, and (3) the two-armed spiral shock wave, and that the $\mathrm{CO}$ was displaced from that place along the line-of-sight having the maximum radial velocity. On these assumptions, the $\mathrm{CO}$ was not found to lie in any large, organized patterns. Specifically, the CO is not found to be exclusively in the spiral arms or in any pattern displaced from, but parallel to, the spiral arms.

Alternatively, the $\mathrm{CO}$ velocities were compared with those of stars whose orbits are perturbed by the gravitational potential in the spiral arms. This model allowed agreement with the CO velocities for stars with "ages" $\leq 30 \times 10^{6}$ years. Some evidence was found that the "age" required for agreement with the $\mathrm{CO}$ velocities increases with galactic longitude through the region observed.
\end{abstract}

Subject headings: galaxies: internal motions - galaxies: structure - interstellar: matter radio sources: $21 \mathrm{~cm}$ radiation

\section{INTRODUCTION}

Observations of the millimeter-wavelength spectral lines of carbon monoxide have primarily concentrated on individual discrete molecular clouds in which these lines are very strong. Recently, several observerse.g., Burton et al. (1975) and Scoville and Solomon (1975) - have conducted surveys of the strength of the carbon monoxide spectral lines in the millimeter wavelength region in the galactic plane over large regions of galactic longitude. Radiation from the $J=1 \rightarrow 0$ transition in ${ }^{12} \mathrm{C}^{16} \mathrm{O}$ isotope (hereinafter called $\mathrm{CO}$ ) at $115.271201 \mathrm{GHz}$ rest frequency is seen at virtually every observed position in the plane, but its strength generally decreases with increasing galactic longitude beyond $l=30^{\circ}$. These survey observations have allowed the above observers to reach conclusions regarding the relative numbers of $\mathrm{CO}$ clouds along the line-of-sight a function of galactic longitude and the mass and temperature of an average cloud.

We have also conducted a survey of the CO spectral line in the galactic plane; however, we shall be primarily concerned with the kinematics of the $\mathrm{CO}$ clouds relative to the predictions of the linear densitywave theory (Yuan 1969a), the two-armed spiral-shock pattern (Shu et al. 1972), and the orbital velocities of stars. The following section discusses the observational data, § III describes the galactic model which was used, \& IV compares the CO kinematics to those predicted for gas in the galactic plane, and the final section compares the radial velocities of the $\mathrm{CO}$ spectral features to those expected for stars orbiting in the galactic plane.

\section{OBSERVATIONS}

The observations were made using the 16 foot $(5 \mathrm{~m})$ millimeter antenna at the Millimeter Wave Observatory of the University of Texas ${ }^{1}$ during the periods 1975 February 14 to 18 and 1975 May 28 to June 2 . The antenna has a half-power beamwidth of 2'.6 at $115 \mathrm{GHz}$, and the receiver double-sideband noisetemperature was approximately $1000 \mathrm{~K}$. The receiver was used with a 40-channel filter bank, each channel of which is $2 \mathrm{MHz}\left(5.2 \mathrm{~km} \mathrm{~s}^{-1}\right)$ wide separated by $2 \mathrm{MHz}$, giving a velocity coverage of $208 \mathrm{~km} \mathrm{~s}^{-1}$.

One observation consists of five two-minute ON-OFF pairs, where the signal from a reference position was subtracted from the signal in the galactic plane. To ensure against the possible presence of a CO spectral line in the reference position, different reference positions were used for each "OFF," all at least $5^{\circ}$ out of the plane, and each one separated by at least $1^{\circ}$ from the others. The spectra displayed in Figure 1 consist of the sum of from three to 10 observations.

We observed at each degree of galactic longitude $(l)$ from $30^{\circ}$ through $60^{\circ}$ at a galactic latitude $(b)$ of zero. The receiver's oscillator was tuned so as to place the central filter of the 40-channel filter-bank at the terminal radial velocity of the $H \mathrm{I}, 21 \mathrm{~cm}$ profile at each longitude. Even though the oN-OFF technique was used, the receiver's stability was not sufficient to

${ }^{1}$ The Millimeter Wave Observatory is operated by the Electrical Engineering Research Laboratory, the University of Texas at Austin, with support from the National Aeronautics and Space Administration, the National Science Foundation, and McDonald Observatory. 

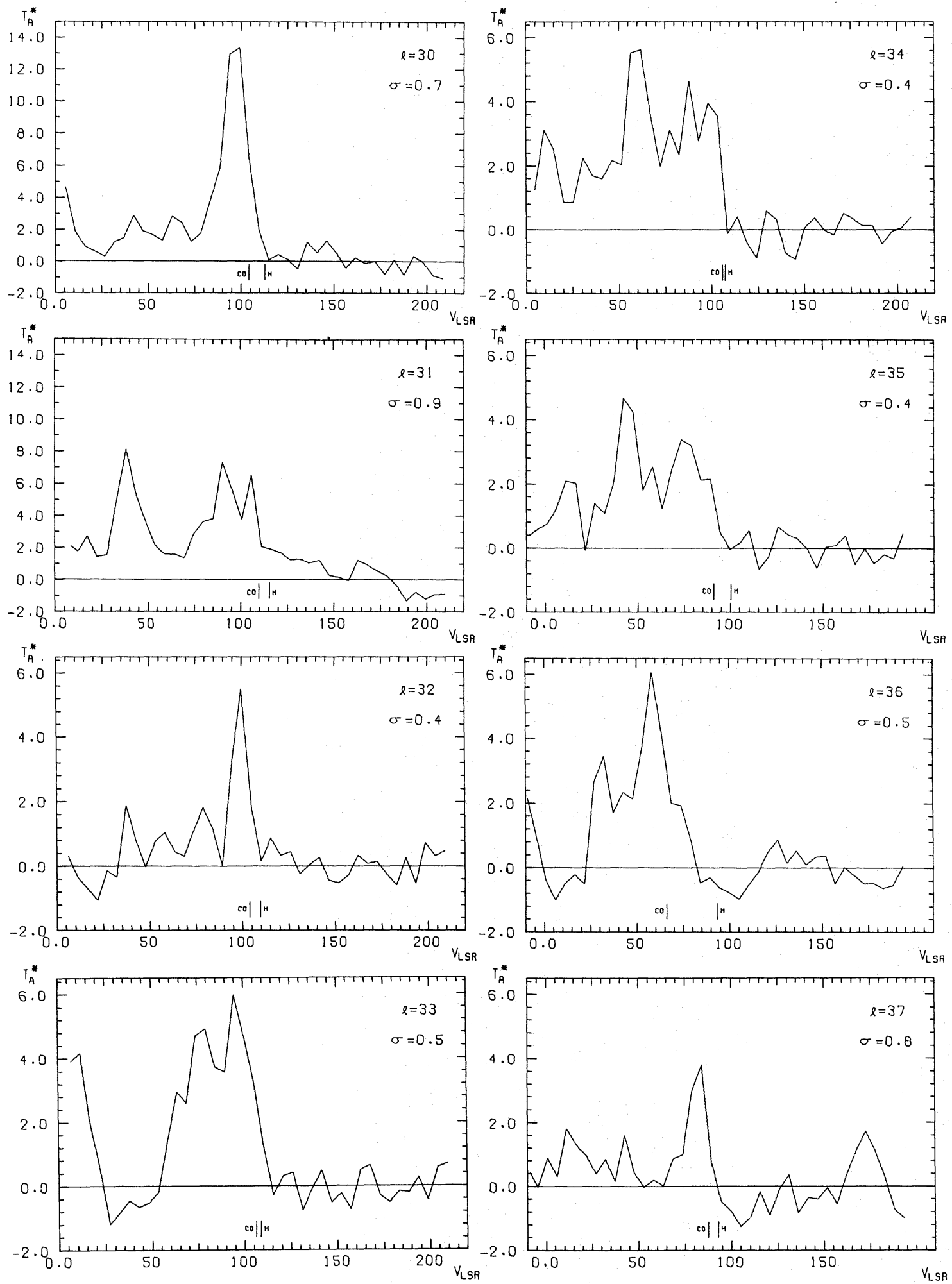

Frg. 1.-The observed CO spectra. The mark "CO" is the profile's terminal velocity, and " $\mathrm{H}$ " is the terminal velocity of the $\mathrm{H} \mathrm{I}, 21 \mathrm{~cm}$ line at the same position. All were observed at $b=0^{\circ}$. The ordinate, $T_{A}{ }^{*}$, is in kelvins, and the radial velocity with respect to the local standard of rest is in kilometers per second. The profiles have been adjusted vertically to force the mean of the fifteen right-most channels to be zero, and the standard deviation of those channels is shown in the upper right. 

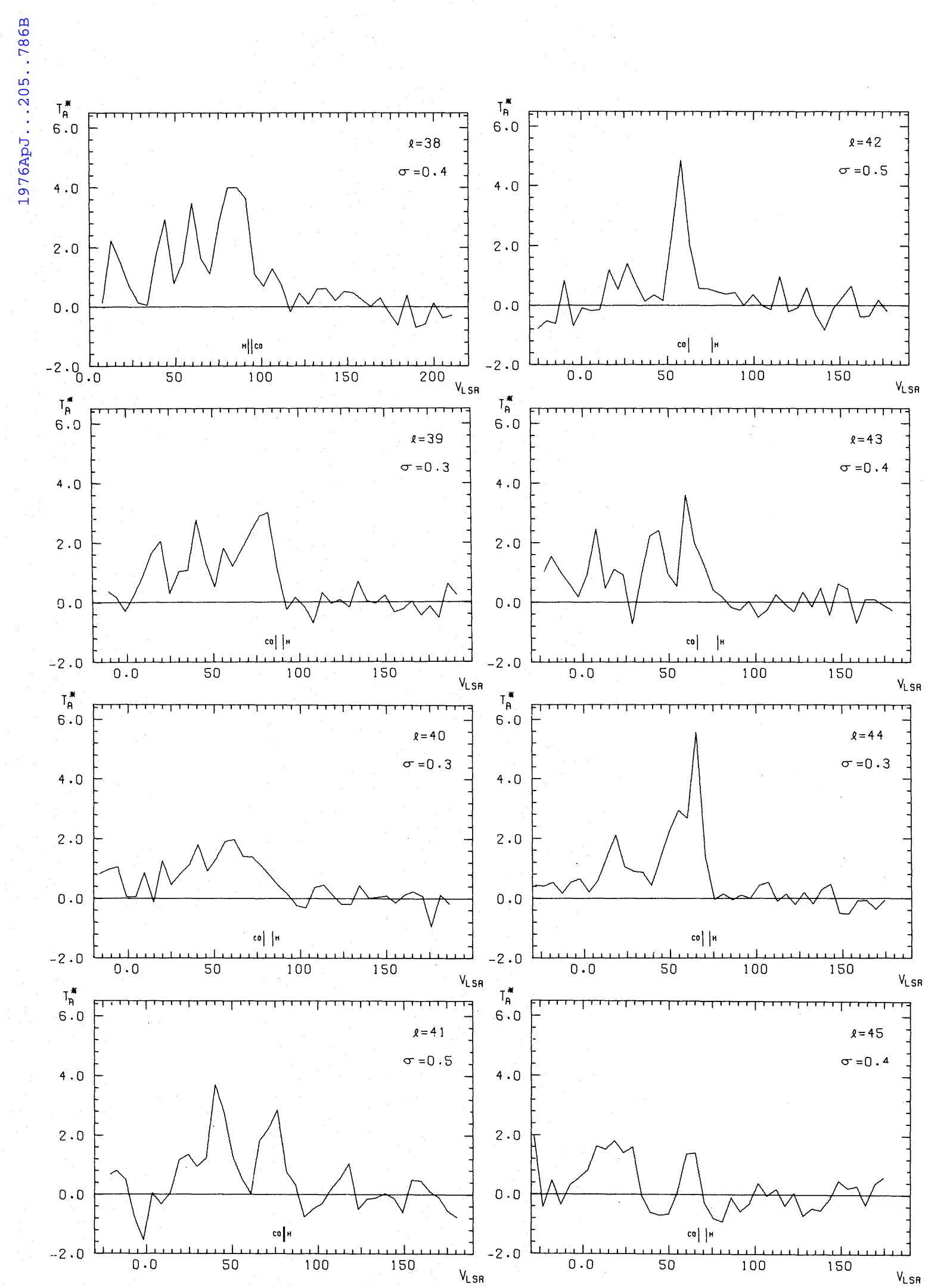

Fig. 1.-Continued 

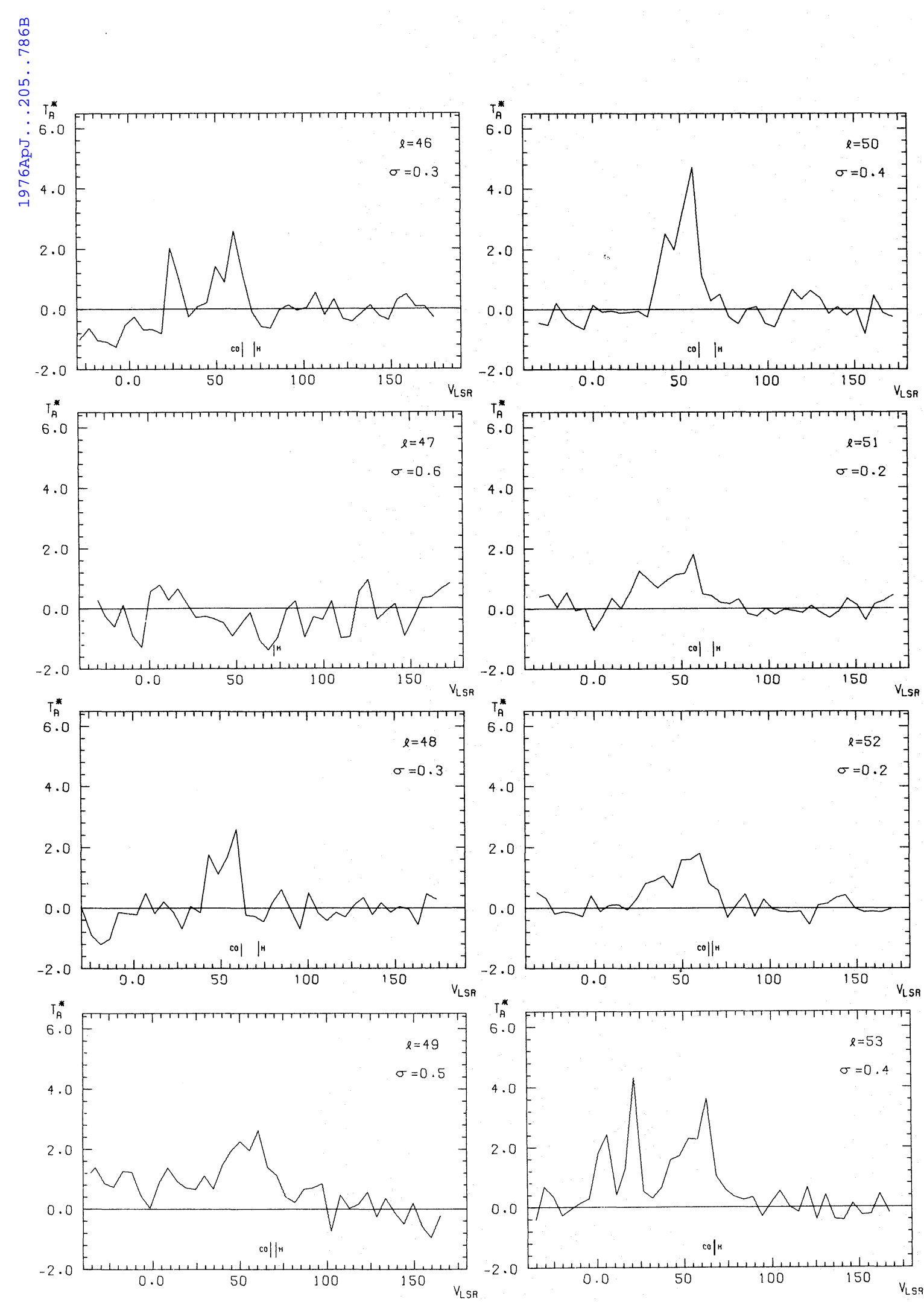

Fig. 1.-Continued 

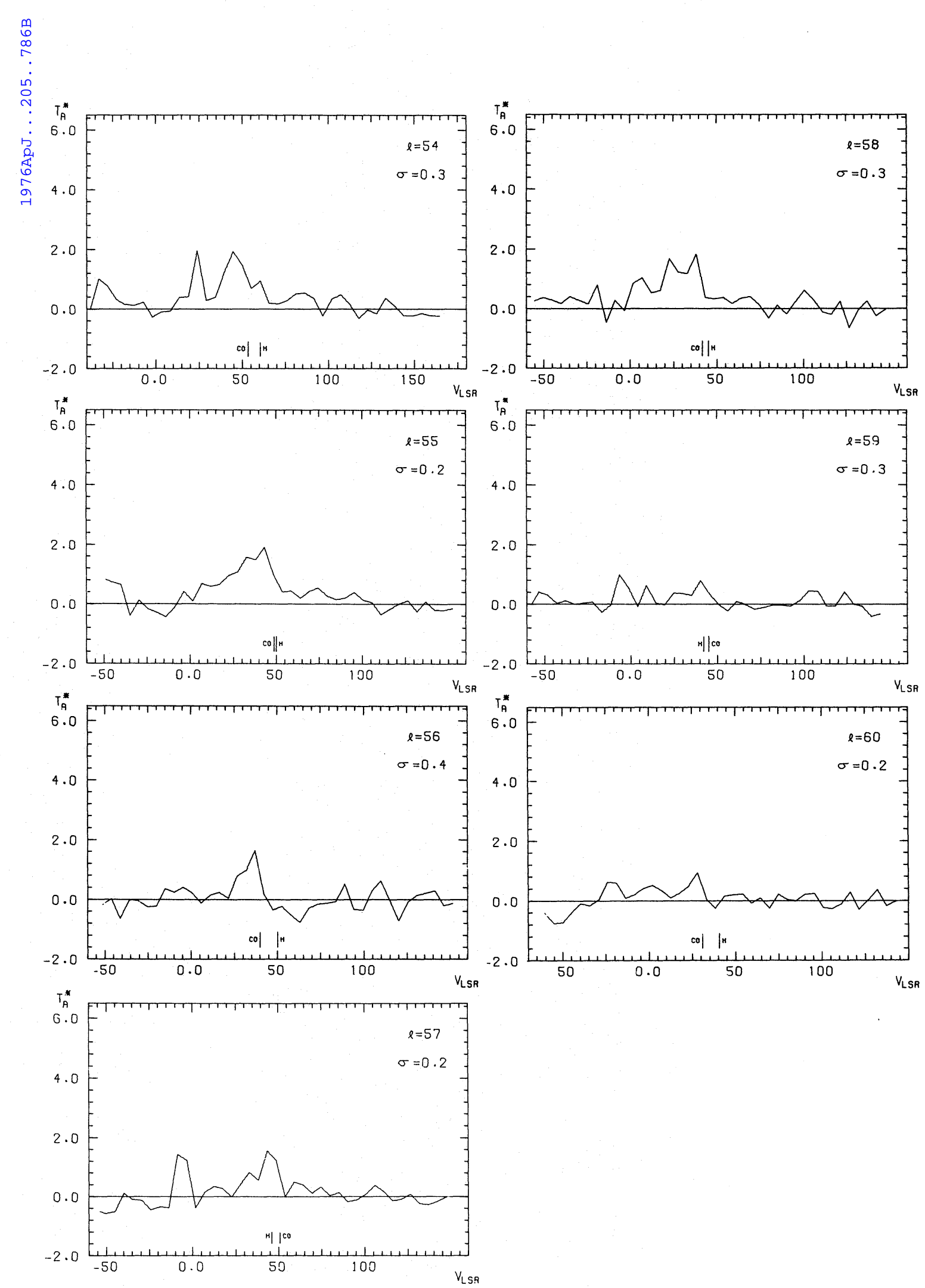

FIG 1.-Continued 
ensure that the baseline was at zero; thus the average antenna temperature of the 15 channels having the highest radial velocity was subtracted from the antenna temperatures in each channel. No smoothing or other baseline removal has been done. This procedure is appropriate so long as no $\mathrm{CO}$ spectral feature occurs farther than $26 \mathrm{~km} \mathrm{~s}^{-1}$ (5 channels) above the $\mathrm{H}$ I profile terminal velocity, and although baseline noise and weak $\mathrm{CO}$ could be confused, no obvious $\mathrm{CO}$ is found in this velocity region.

Figure 1 shows the $\mathrm{CO}$ profile at each longitude. The ordinate is corrected antenna temperature, denoted $T_{A}{ }^{*}$, in kelvins. This quantity gives the intensity in the line in units of radiation temperature $\left[\Delta J\left(T_{\mathrm{LE}}\right)\right.$ of Zuckerman and Palmer 1974] corrected for atmospheric attenuation and antenna losses and assuming that the source fills the beam. The abscissa is the radial velocity with respect to the local standard of rest in $\mathrm{km} \mathrm{s}^{-1}$. Each panel includes the standard deviation of the population of baseline samples expressed in kelvins. The mark labeled " $\mathrm{CO}$ " is the $\mathrm{CO}$ profile's terminal velocity, and the mark " $\mathrm{H}$ " is the H I, $21 \mathrm{~cm}$ terminal velocity at the same longitude. The terminal velocity $\left(V_{T}\right)$ is here defined as the velocity at which the high-velocity end of the line profile reaches half its height. This particular measure is somewhat noisy in that it depends on only two or three points; however, it is quite insensitive to the baseline noise. The $\mathrm{H} \mathrm{I}, 21 \mathrm{~cm}$ terminal velocities were computed from the Westerhout (1969) survey data. As the signal-to-noise ratio and the velocity resolution of the $\mathrm{H}$ I data is much greater than that of the CO data, the uncertainty in the value of the terminal velocity of the $\mathrm{HI}$ is much less than that of the CO.

Figure 2 shows the terminal velocity difference $V_{T}(\mathrm{CO})-V_{T}(\mathrm{H} \mathrm{I})$ as a function of galactic longitude. Uncertainties arise in this difference due to $(a)$ choices of "peaks" or "shoulders" involved in computing $V_{T}$, (b) noise in the profiles, and (c) the rather large width of the filters which we used. An examination of Table 1

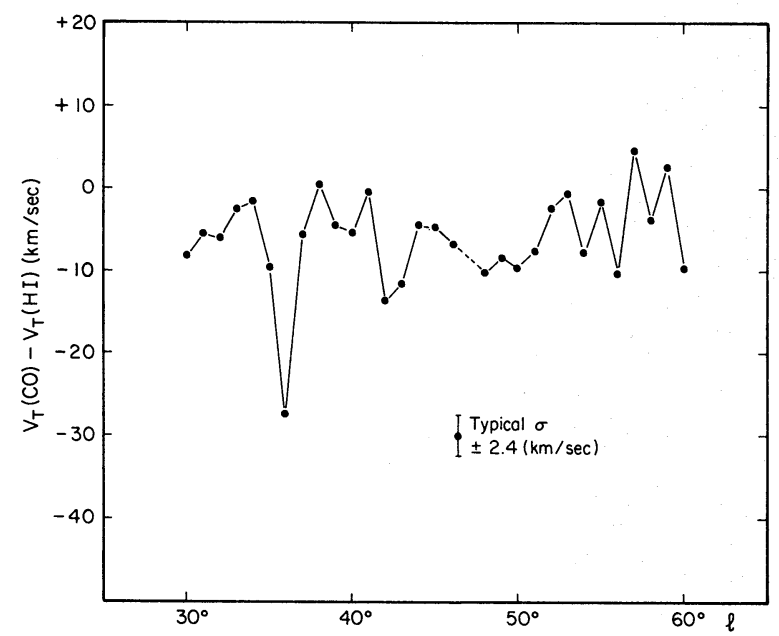

FIG. 2.-The difference between the $\mathrm{CO}$ and $\mathrm{H}$ I terminal velocities as a function of galactic longitude. The expected standard deviation of each point is $2.4 \mathrm{~km} \mathrm{~s}^{-1}$.

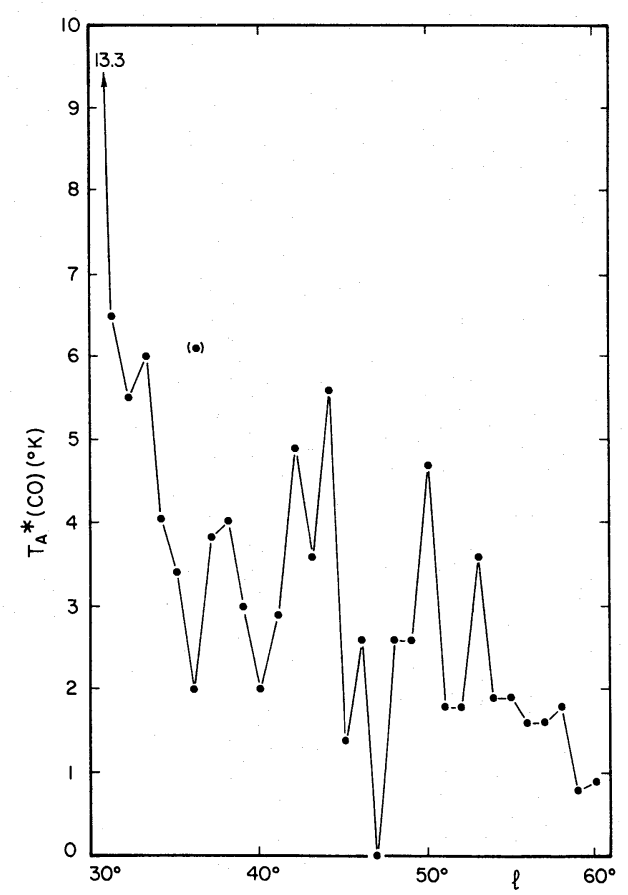

FIG. 3.-The height of the terminal feature of the $\mathrm{CO}$ profiles as a function of galactic longitude. Especially at $l=36^{\circ}$, confusion arises due to a shoulder on the peak, and both points are shown. No CO was detected at $l=47^{\circ}$.

of Shane and Bieger-Smith (1966) for $\mathrm{H}$ I profiles over the same longitude range gives $\sigma\left(V_{T}\right) \approx 1.2 \mathrm{~km} \mathrm{~s}^{-1}$ for cause $(a)$. Numerical experiments on the $\mathrm{CO}$ data in which the height of the peak at the positive velocity end of the profile was moved up and down by 1.4 times the baseline standard deviation give $1.0 \mathrm{~km} \mathrm{~s}^{-1}$ for cause $(b)$, and we assign 0.7 of the filter half-width, $1.8 \mathrm{~km} \mathrm{~s}^{-1}$, to cause $(c)$. This gives an expected standard deviation of the difference $V_{T}(\mathrm{CO})-V_{T}(\mathrm{H} \mathrm{I})$ of $2.4 \mathrm{~km} \mathrm{~s}^{-1}$. Therefore, significant negative differences occur at longitudes of $36^{\circ}$ and $42^{\circ}$, and the average difference is significantly less than zero. This average negative difference is physically significant only if the velocity dispersions at the terminal ends of both the $\mathrm{H}$ I and $\mathrm{CO}$ profiles are equal. Otherwise, the negative difference is due to different velocity distances between $\mathrm{H} I$ and $\mathrm{CO}$ from the terminal cloud's centroid velocity to the half-height velocity. We did not detect any $\mathrm{CO}$ at $l=47^{\circ}$.

Figure 3 displays the temperature, $T_{A}{ }^{*}$, of the $\mathrm{CO}$ terminal feature plotted against $l$. This temperature drops rapidly with increasing $l$.

\section{GALACTIC MODEL}

The subsequent two sections involve the predictions of a model of the kinematics in the Galaxy. We adopt a cylindrical coordinate system centered on the galactic center. If the Galaxy is viewed from the north galactic pole, it rotates clockwise, and the azimuth angle $\theta$ increases clockwise from the radius which passes 
through the Sun at the present instant of time. The radial coordinate is $\varpi$.

The linear density wave model of Yuan (1969a) has been used with the pitch angle, $i=-6: 65$, and the number of arms, $M=2$. Arms cross the $\theta=0$ line at $\varpi=5.62 \mathrm{kpc}, 8.11 \mathrm{kpc}$, and $11.70 \mathrm{kpc}$, and the radial phase term $\Phi(\varpi)$ is approximated by a logarithmic spiral. The pattern speed $\Omega_{p}$ is assumed to be $13.5 \mathrm{~km} \mathrm{~s}^{-1} \mathrm{kpc}^{-1}$ with respect to an external inertial frame. The angular velocity of galactic rotation, $\Omega$, is taken from the force function $\varpi \Omega^{2}$ used by Contopoulos and Strömgren (1965) in which the angular velocity at the Sun is $\Omega_{0}=25 \mathrm{~km} \mathrm{~s}^{-1} \mathrm{kpc}^{-1}$ and the Sun lies at $\varpi_{0}=10 \mathrm{kpc}$ from the galactic center. This function fits the Schmidt (1965) rotation curve extremely well over our range of interest.

\section{GAS KINEMATICS}

In this section we shall attempt to interpret the kinematics of the terminal portion of the $\mathrm{CO}$ profile on the assumption that galactic $\mathrm{CO}$ behaves like a gas which has the same kinematics as the interstellar $\mathrm{H}$. By using the velocity difference shown in Figure 2, between the $\mathrm{CO}$ and $\mathrm{H}$ I terminal velocities, uncertainties in the actual rotation curve do not cause first-order effects. If one assumes that interstellar $\mathrm{H} \mathrm{I}$ is ubiquitous, every detail of the galactic velocity curve is mapped into the $\mathrm{H} \mathrm{I}, 21 \mathrm{~cm}$ line profile. Whereas negative values of $V_{T}(\mathrm{CO})-V_{T}(\mathrm{H} \mathrm{I})$ in Figure 2 are understandable, positive values are forbidden. Negative values of the difference arise if $\mathrm{CO}$ is displaced from the place along the line-of-sight where the $\mathrm{H}$ I has its maximum radial velocity. Positive values could only occur if, at the maximum radial velocity position, no $\mathrm{H} \mathbf{I}$ is present.

Velocity differences between the $\mathrm{CO}$ and $\mathrm{H}$ I terminal velocities can be converted to distance difference along the line-of-sight if one has a model for the galactic kinematics. We shall now attempt such an interpretation assuming $(a)$ pure circular rotation, $(b)$ the linear density wave impressed on the galactic rotation, and (c) the two-armed spiral shock pattern.

\section{a) Pure Circular Rotation}

In pure circular rotation where $d \Omega(\varpi) / d w<0$ (as in the Schmidt rotation curve), the maximum radial velocity occurs at the subcentral point (the place along the line-of-sight closest to the galactic center), and $\varpi_{\text {scp }}=\varpi_{0} \sin l$. In addition, the radial velocity function along the line-of-sight and inside the $\varpi=\varpi_{0}$ circle is symmetric about the subcentral point. Thus, if $\mathrm{CO}$ is seen at a lower velocity than the $\mathrm{HI}$, the $\mathrm{CO}$ source may lie at either of two places symmetric about the subcentral point. Figure 4 shows the displacement along the line-of-sight $\left(r_{\mathrm{Co}}\right)$ required to explain Figure 2 on the assumption of pure circular rotation. The curve in Figure 4 marked " + " is the distance from the subcentral point away from the Sun. The curve marked "-" is the corresponding distance toward the Sun. Within this distance of the subcentral point there must be no detectable $\mathrm{CO}$ clouds. The dotted line

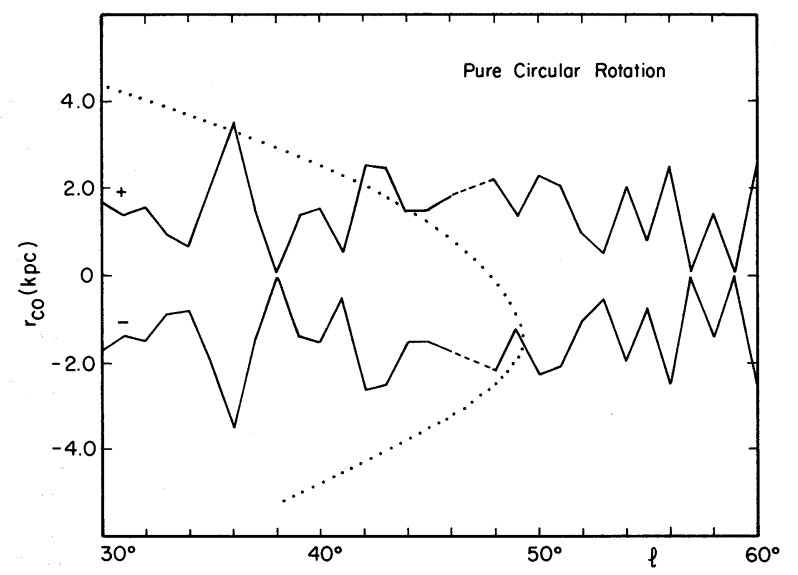

FIG. 4.-For pure circular rotation, the displacement from the subcentral point either away from the Sun $(+)$ or toward the Sun $(-)$ required to explain the observations shown in Fig. 2. For comparison, the distance from the subcentral point to our model's position for the Sagittarius arm is shown as the dotted line.

shows the displacement from the subcentral point to the "Sagittarius arm." Notice that the "Sagittarius arm" in the model is tangent to the line-of-sight at $l \approx 49^{\circ}$. Inside of that longitude, the line-of-sight cuts across that arm twice. The distance to the arm is not symmetric about zero mainly because the locus of subcentral points is a circle of radius $\varpi_{0} / 2$ centered on $\varpi_{0} / 2, \theta=0^{\circ}$, and secondarily because the arms are spirals. At $l=37^{\circ}, 58^{\circ}$, and $59^{\circ}$ the velocity difference in Figure 2 becomes slightly positive. If this were significant, the $\mathrm{H}$ I would be displaced from the subcentral point instead of the CO. For these points, $r_{\mathrm{Co}}$ was set to zero.

If the CO exists as interstellar gas which is optically thin or if it is in the form of a large number of optically thick clouds, each much smaller than the antenna beam, the height of the terminal velocity portion of the $\mathrm{CO}$ profile is related to the velocity gradient near the subcentral point. Figure 5 gives the length along the line-of-sight, centered on the subcentral point $(\Delta r)$ over which the radial velocity varies by $2.5 \mathrm{~km} \mathrm{~s}^{-1}$. One can see that this smoothly varies with $l$. This figure is included because it measures the gradient of radial velocity along the line-of-sight at the highest radial velocity along it. If $\mathrm{CO}$ were optically thin, its number density, at this highest velocity point, is related to the height of the terminal portion of the profile, $T_{A}^{*}$, divided by $\Delta r$.

\section{b) Linear Density Wave}

The equations of Yuan (1969a) were used (after correcting some errors in them) to predict the effects of the linear density wave. The amplitudes of the radial and tangential velocity components were assumed to be $8 \mathrm{~km} \mathrm{~s}^{-1}$, the same value that Yuan (1969a) used to fit the $\mathrm{H}_{\mathrm{I}}, 21 \mathrm{~cm}$ rotation curve. Here, the maximum radial velocity does not necessarily fall at the subcentral point, and the radial velocity run along the 


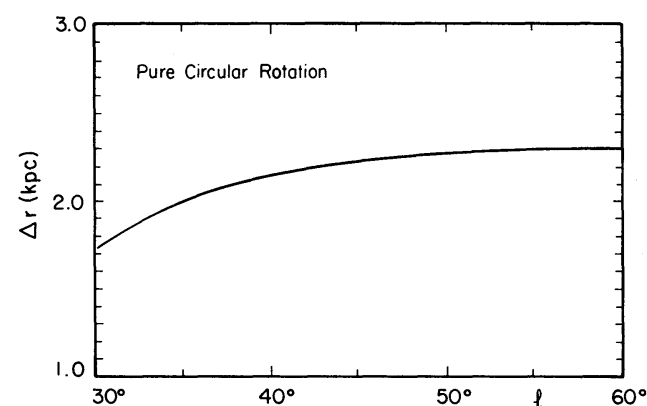

FIG. 5.-For pure circular rotation, the distance range, centered on the subcentral point velocity, over which the velocity changes by $2.5 \mathrm{~km} \mathrm{~s}^{-1}$. This distance is the sum of the displacement toward the Sun and away from the Sun.

line-of-sight is not symmetric about it. Figure 6 shows the distance along the line-of-sight by which the gas must be displaced from the subcentral point to explain Figure 2. The highest velocity point is displaced from the subcentral point by as much as $2 \mathrm{kpc}$. Figure 7 shows the distance along the line-of-sight, centered on the highest velocity point, over which the velocity changes by $2.5 \mathrm{~km} \mathrm{~s}^{-1}$.

\section{c) Two-armed Spiral Shock}

The peculiar velocities caused by the galactic twoarmed spiral shock wave (TASS) were taken from Figure 2 of Shu et al. (1972), where the ratio of the radial component of the spiral gravitational field to the smooth background field, $F=0.05$. This peculiar velocity field was assumed for all the spiral arms, and the shock was placed $37^{\circ}$ of phase inside the centers of the spiral arms. These two assumptions are somewhat crude. The highest velocity point is now found to be displaced by as much as $3.5 \mathrm{kpc}$ from the subcentral

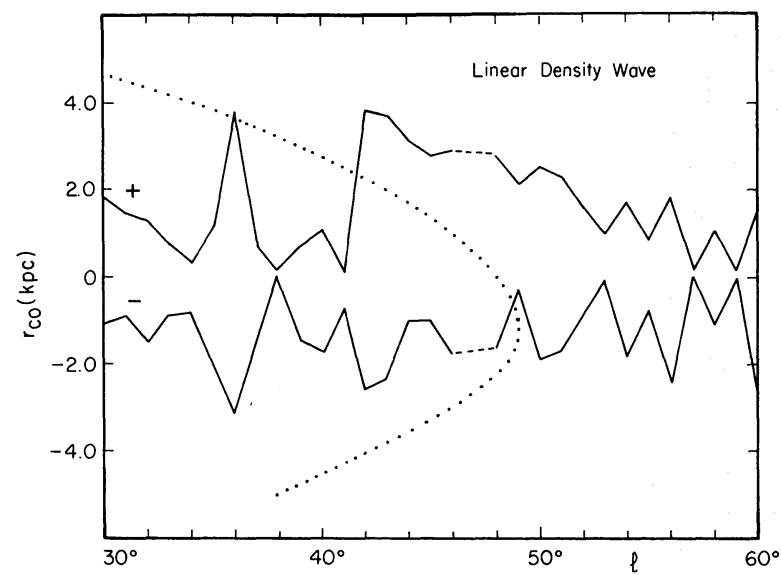

FIG. 6.-For the linear density wave, the displacement from the subcentral point either away from the Sun $(+)$ or toward the Sun $(-)$ required to explain the observations shown in Fig. 2. For comparison, the distance from the subcentral point to our model's position for the Sagittarius arm is shown as the dotted line.

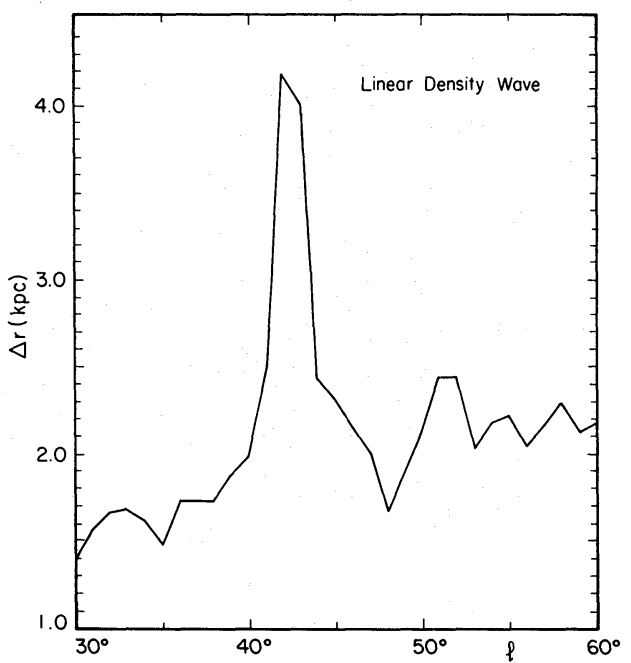

FIG. 7.-For the linear density wave, the distance range, centered on the highest radial velocity point, over which the velocity changes by $2.5 \mathrm{~km} \mathrm{~s}^{-1}$. This distance is the sum of the displacement toward the Sun and away from the Sun.

point, and quite steep velocity gradients are found along the line-of-sight near the shock wave. Figure 8 shows the amount by which the $\mathrm{CO}$ needs to be displaced from the subcentral point where " + " and "-" have the same meaning as in Figure 6. Figure 9 shows the distance along the line-of-sight centered on the highest velocity point over which the velocity changes by $5 \mathrm{~km} \mathrm{~s}^{-1}$.

Examining Figures 4, 6, and 8, one does not see any obvious pattern of $\mathrm{CO}$ displacement along the line-ofsight relative to the position of the spiral arm. If the $\mathrm{CO}$ is exclusively concentrated along spiral arms, one

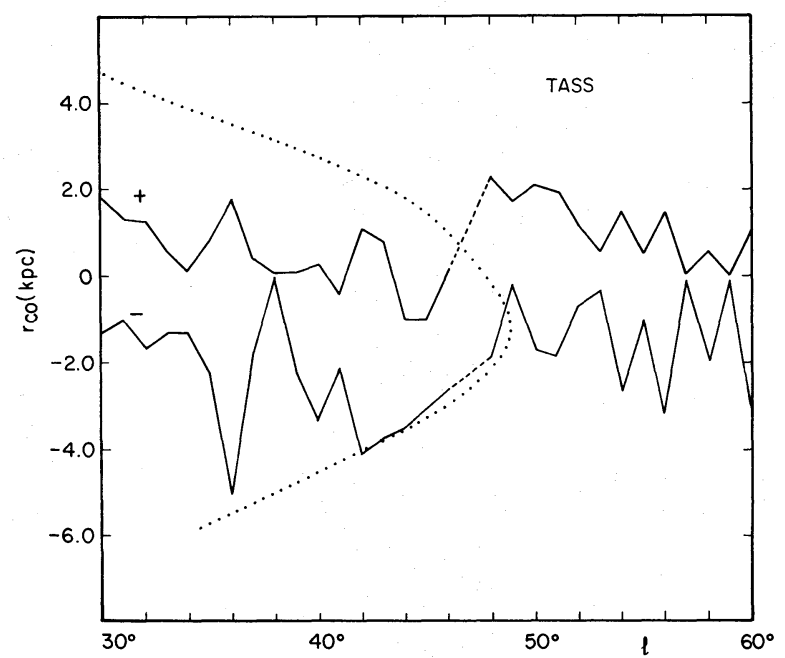

FIG. 8--For the two-armed spiral shock wave (TASS), the displacement from the subcentral point either away from the Sun $(+)$ or toward the Sun $(-)$ required to explain the observations shown in Fig. 2. For comparison, the distance from the subcentral point to our model's position for the Sagittarius arm is shown as the dotted line. 


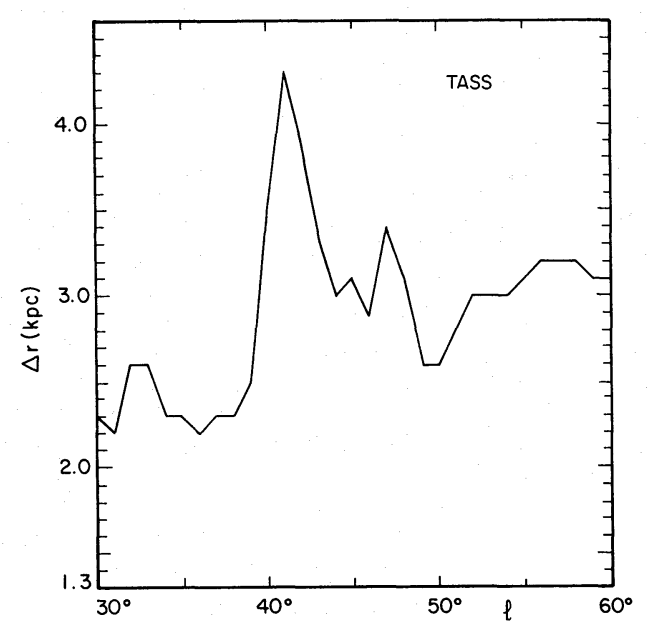

FIG. 9.-For the two-armed spiral shock wave (TASS), the distance range, centered on the highest radial velocity point, over which the velocity changes by $5.0 \mathrm{~km} \mathrm{~s}^{-1}$. This distance is the sum of the displacement toward the Sun and away from the Sun.

should expect the $r_{\mathrm{co}}$ to tend to decrease with $l$ as one approached the tangent point of the spiral arm, then suddenly increase where the line-of-sight misses the spiral arm entirely. The pure circular rotation case (Fig. 4) shows no such effect, and for longitudes $42^{\circ}-50^{\circ}$, large portions of the lines-of-sight lying within the spiral arm must be deficient in molecular clouds. The linear density wave case (Fig. 6) has the same problem for these longitudes. There is possibly such a decrease, then a sudden increase, then a decrease pattern, but the discontinuity in Figure 8 is near $l=40^{\circ}$ instead of $l=50^{\circ}$ where the arm is tangent to the line-of-sight. The TASS model (Fig. 8) shows a slight decrease toward $l=50^{\circ}$, but it is not followed by the large increase expected. The CO appears to run along the spiral arm over longitudes $42^{\circ}-50^{\circ}$, and the TASS model is therefore considered to be a better fit to the data than the other models, but inside $l=42^{\circ}$ the $\mathrm{CO}$ no longer fits the arm.

One of the especially significant pieces of evidence regarding the hypothesis that the $\mathrm{CO}$ lies in or near the spiral arms is found in the region $l \approx 52^{\circ}$ to $60^{\circ}$. In this longitude range, the next outer spiral arm is the Perseus arm which lies beyond $w=10 \mathrm{kpc}$ in this region. Thus, if the $\mathrm{CO}$ lies in or near that arm, one should see at least a $50 \mathrm{~km} \mathrm{~s}^{-1}$ velocity jump near $l=50^{\circ}$ which persists to $l=60^{\circ}$.

Thus we conclude that one cannot rule out, on the basis of these data, the hypothesis that the CO clouds have the same kinematics as atomic hydrogen but simply are not as uniformly distributed in the Galaxy as the hydrogen. However, we find no evidence that this nonuniformity is related to an exclusive concentration of the CO clouds in spiral arms, or in any pattern displaced from but parallel to the spiral arms. It should be emphasized that the preceding point is true even if one believes that no velocity difference in Figure 2 is significantly different from zero. If that were the case, then one would have to conclude that the $\mathrm{CO}$ exists at each point along each line-of-sight where $\mathrm{H}$ I has its maximum radial velocity, including those points between spiral arms.

\section{STAR DYNAMICS}

The association of $\mathrm{CO}$ molecular clouds with young stars and regions of star formation suggests that these molecular clouds might be gravitationally bound, and exhibit dynamics similar to stars or clusters of stars. If so, the $\mathrm{CO}$ molecular clouds may be expected to have different kinematics from the background $\mathrm{H}$ I even in the same region of the Galaxy. In the case of stellar dynamics, one can entirely neglect pressure forces due to cloud-cloud or cloud-gas collisions. This alternative is reasonable if one takes a "typical cloud" having a diameter of $1 \mathrm{pc}$ and a molecular hydrogen density of $10^{3} \mathrm{~cm}^{-3}$ moving at $10 \mathrm{~km} \mathrm{~s}^{-1}$ through $\mathrm{H} \mathrm{I}$ of density $1 \mathrm{~cm}^{-3}$. It will take $10^{8}$ years for the cloud to sweep out a tube containing a mass equal to its own mass. If the cloud is collapsing, this time is lengthened. Thus, on time scales less than this, the motion of the cloud will be similar to that of a star. In this sense we will refer to the molecular cloud as a "star" throughout the following discussion, although we shall be computing the ballistic trajectories of particles moving solely under the influence of gravity.

To investigate this alternative, stellar orbits were computed in an attempt to associate the stellar velocities and positions with the velocities of the $\mathrm{CO}$ terminal features. Stellar orbits were computed in the Galaxy, and the perturbation of the spiral arms was taken into account. The initial velocities were those predicted for the shock wave on the inside edge of the spiral arms. The equations given by Yuan $(1969 b)$ were integrated (after correcting some errors) ${ }^{2}$ using a Runge-Kutta numerical integrator; 0.05 times the background potential was assumed to be in the spiral pattern, and the pattern described in § III was used. The stars were assumed to be born only on the inside edge of the spiral arms $-0.21 \mathrm{kpc}\left(30^{\circ}\right.$ of radial phase) from the arm center. The initial star velocities were assumed to be those of the TASS shock wave given in Figure 2 of Shu et al. (1972).

The model assumed 36 sites of star birth and that at each, six stars were born each $10^{6}$ years. These sites are spaced $10^{\circ}$ apart in $\theta$ from $\theta=-90^{\circ}$ to $+90^{\circ}$, and lie on the inside edges of the two spiral arms which cross between the Sun and the galactic center. The six stars born at each site sample the velocities through the shock wave and introduce an initial velocity dispersion. The six initial velocities, $\left(V_{\xi}, V_{\eta}\right)=$ (109., 2.5), (101., 2.5), (109., 25.5), (101., 25.5), (93., 2.5), and (93., 25.5) $\mathrm{km} \mathrm{s}^{-1}$, were taken from Figure 2 of Shu et al., where they express the shock wave velocities in a rotating coordinate system in which $\xi$ is parallel

${ }^{2}$ In Yuan (1969b) equations (5) and (7) should read $d \Psi / d T$ instead of $d \Psi / d t$, the right-hand term of equation (7) should have a minus sign, and the sign between the two right-hand terms in the second line of the definition of $\Delta$ should read " + " instead of "-.". 
to the spiral arms and $\eta$ is perpendicular to them. The model assumed that the stars are all born at the current instant, and the orbit of each of the 216 stars was integrated over 100 time steps, each of which is $10^{6}$ years. At the end of each time step, each star's position $(\varpi, \theta)$ and linear velocity in the two orthogonal directions $\left(V_{\theta}, V_{w}\right)$ was computed.

To investigate the current positions and velocities of stars born over the last $10^{8}$ years, the positions of the stars were rotated in $\theta$. That is, the current position and velocity of stars of age zero is associated with time step zero-the birth place and initial velocities. The stars which are currently, say, $10^{6}$ years old were born when the spiral pattern was rotated back by $\Omega_{p} \times 10^{6}$ years, and the current position of a star born $10^{6}$ years ago is the result of the numerical integration after the first time step with $\theta$ rotated back by $\Omega_{p} \times 10^{6}$ years, and so on. Thus we have 21,600 stars ranging in age from 0 to $10^{8}$ years. Finally, the list was searched to find all the stars within $10^{\prime}$ of each of the lines-of-sight which were observed and at each line-of-sight the stars were sorted into decreasing order of radial velocity with respect to the local standard of rest. Each star is described by its present galactic longitude, distance from the Sun, radial velocity, birth place, initial velocity, and age. This age is not a conventional stellar age, but begins when the protostellar cloud becomes very dense, presumably after passing through the shock front, and can be regarded thereafter as a ballistic particle.

The general results of the model computations will first be described. The stars found at each longitude, over the range $30 \leq l \leq 60^{\circ}$, show that (1) the older stars have the highest radial velocities, (2) the highest radial velocities found considerably exceed the $\mathrm{CO}$ terminal velocity, (3) no star whose age is less than $73 \times 10^{6}$ years occurs at $l \geq 47^{\circ}$, and (4) the fraction of the total number whose age is $\leq 30 \times 10^{6}$ years varies from approximately 0.1 between $30^{\circ}$ and $35^{\circ}$, to 0.15 between $35^{\circ}$ and $45^{\circ}$, to zero beyond $47^{\circ}$.

The line-of-sight is currently tangent to the inner spiral arms at $l=30^{\circ}$ and $l=49^{\circ}$, and each birth site is about $2^{\circ}$ inside of this tangent place. The initial velocities cause all the stars to have no inward movement with respect to their birth place for at least $10^{6}$ years and a maximum outward motion of about $0.25 \mathrm{kpc}$ in the first $10^{6}$ years. The fact that only old stars are seen between the arms derives from our assumption that stars are born in the arms.

When one looks down the lists of stars which the model generates at each longitude, as one encounters lower radial velocities, one also finds younger stars. Figure 10 shows the radial velocity of the first star encountered whose age is less than or equal to $35 \times 10^{6}, 30 \times 10^{6}$, and $25 \times 10^{6}$ years. That is, these stars are the ones with the highest radial velocity and with ages below the limit. No stars this young occur beyond $l=47^{\circ}$, and no stars at all are found at $l=59^{\circ}$ and $60^{\circ}$.

Figure 11 compares the data shown in Figure 2 with the stellar velocities for ages $\leq 30 \times 10^{6}$ years, since those stars show the best agreement with the observa-

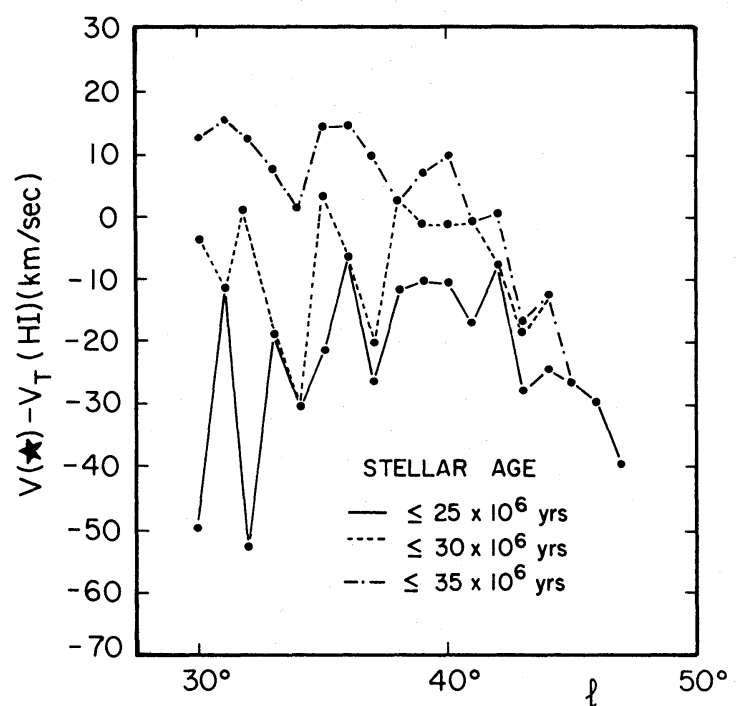

FIG. 10.-The radial velocities of stars from the model minus the terminal velocities of the $\mathrm{H} \mathrm{I}, 21 \mathrm{~cm}$ line for stars of three age groups. Beyond $47^{\circ}$, the youngest star is $73 \times 10^{6}$ years old.

tions. Beyond $l=47^{\circ}$, the radial velocity of the youngest star is plotted, and all stellar ages exceed $73 \times 10^{6}$ years there. The star which has the highest radial velocity along each line-of-sight in the $\leq 30 \times$ $10^{6}$ age group (1) has a similar radial velocity to the $\mathrm{CO}$ terminal velocity, (2) shows some small positive velocity differences as compared with the $\mathrm{H}$ I, (3) shows considerable velocity changes in the vicinity of $l=35^{\circ}$ where the $\mathrm{CO}$ velocity shows a large drop, (4) agrees significantly better with the $\mathrm{CO}$ observations than slightly older or slightly younger stars, (5) has an age $\geq 20 \times 10^{6}$ years, and (6) shows a velocity drop in the vicinity of $l=45^{\circ}$ which the observations do not show.

The computer model allows agreement with the observations if we associate ages of $20-30 \times 10^{6}$ years with the terminal end of the profile. The velocity

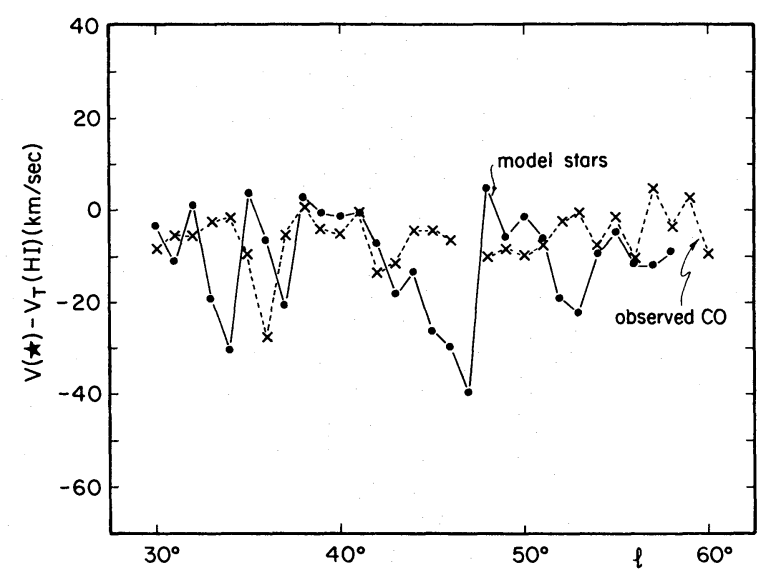

FIG. 11.-The observations in Fig. 2 compared with the stars whose ages are $\leq 30 \times 10^{6}$ years from Fig. 10 . Beyond $l=47^{\circ}$, the radial velocity of the youngest star at each galactic longitude is shown. 
components found at lower radial velocities along the $\mathrm{CO}$ profile occur at the radial velocities of still younger stars. Again, if one were to suggest that no velocity difference in Figure 2 were significantly different from zero, the stellar age which agrees with the CO observations would be increased only slightly.

Although this model for stellar births and stellar orbits shows some agreement between stars in a certain age range and $\mathrm{CO}$ velocities, one does not know how sensitive the agreement is to details of the model. However, most of the parameters of the model have been determined by a comparison with $\mathrm{H}$ I kinematics or stars by, e.g., Yuan $(1969 a, 1969 b)$, and are presumably known with fair accuracy or at least allow agreement with other observations.

Perhaps the most uncertain parameter is the position of star births with respect to the spiral arms. To test this, the galaxy model was left unchanged, but the position of star births was moved with respect to the inside edge of the arms in the radial direction, first outward by $1 \mathrm{kpc}$, then inward by $1 \mathrm{kpc}$.

The result of these two perturbations was that $(a)$ in general, stars whose ages $\leq 30 \times 10^{6}$ years still show the best general agreement but not nearly as good as the unperturbed model, $(b)$ when the stellar birthplace is moved out by $1 \mathrm{kpc}$, young stars are found out to $l=60^{\circ}$, but the star velocities are much too small in the vicinity of $l=55^{\circ},(c)$ when it is moved inward by $1 \mathrm{kpc}$, young stars extend out to only $37^{\circ}$, and the stellar velocities are much too small near $l=43^{\circ}$, with no stars beyond $l=53^{\circ}$.

Many uncertainties remain which may affect the value of the "age" which we deduced above. For example, our placement of the shock front differs very slightly $(\sim 0.04 \mathrm{kpc})$ from that in Shu et al. (1972). Shu, Milione, and Roberts (1973) investigate the shock wave behavior inside of $\varpi=10 \mathrm{kpc}$ and find that the shock wave's velocities and its placement with respect to the arms differ from the values at $\varpi=10 \mathrm{kpc}$. This may have a very small effect in this work, however, since all the stars which match the $\mathrm{CO}$ velocities turn out to have been born in the Sagittarius arm. However, before elaborating our model further we wish to observe a sequence of open clusters and associations having main-sequence turnoff ages ranging up to $10^{8}$ years for the presence or absence of $\mathrm{CO}$ so that changes in the model bring it into agreement with the observations.

An additional feature of this model comes from an attempt to explain the amplitudes of the terminal features of the $\mathrm{CO}$ profiles shown in Figure 3. If stars in the age range 20-30 million years are associated with the terminal portion of the $\mathrm{CO}$ profile inside $l=47^{\circ}$, then the numbers of these stars, per unit of radial velocity, at various lines-of-sight might be related to the amplitude of this terminal position. We have used the model to calculate the fraction of the total number of stars along each line-of-sight which are in the age range $20-30$ million years and within $50 \mathrm{~km} \mathrm{~s}^{-1}$ of the highest radial velocity star. We then assume that the height of the terminal portion of the CO profile minus $2.0 \mathrm{~K}\left(T_{A}{ }^{*}-2.0 \mathrm{~K}\right)$ is related to the total number of stars at each line-of-sight times this fraction. $\left(T_{A}^{*} \approx 2.0 \mathrm{~K}\right.$ beyond $l=47^{\circ}$ where the fraction is zero.) If the current number of stars along each line-of-sight varies as $\exp \left(-2.0 \varpi_{\mathrm{scp}}\right)\left(\varpi_{\mathrm{scp}}=\right.$ $\varpi_{0} \sin l$ ) across the region we observed, then the above fraction times the number gives the dashed curve in Figure 12. The curve shown in Figure 3 is also shown for comparison. The fraction as a function of $l$ is shown at the bottom of Figure 3 .

Figure 12 shows reasonable agreement between this very simple model and the observations. Of course, no explanation is included for the fact that $\mathrm{CO}$ is seen beyond $l=47^{\circ}$, yet there are no stars whose ages are less than $73 \times 10^{6}$ years.

We are not unaware of the fact that the dynamical model allows us to connect the numbers of stars and their positions now, with the numbers and positions at birth. Such work will follow in a later paper.

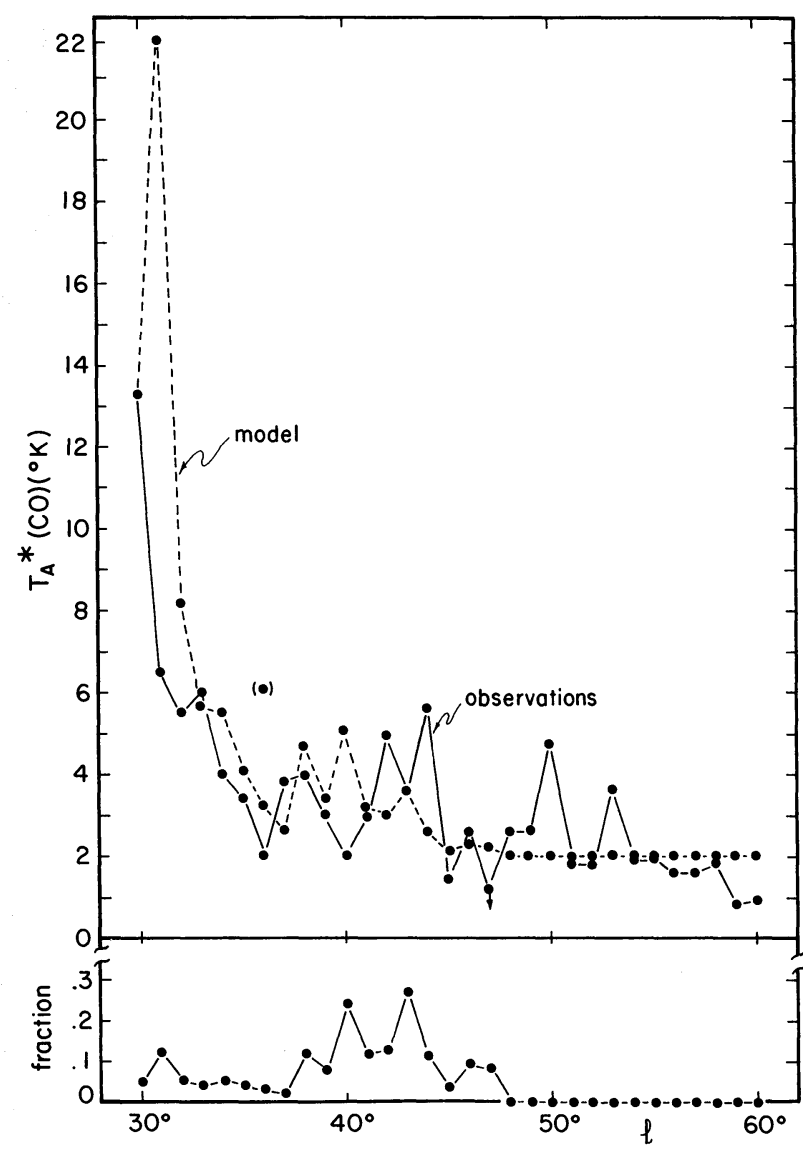

FIG. 12.-The observed height of the terminal portion of the $\mathrm{CO}$ profile from Fig. 3 is compared with the predictions of a model (upper panel). The model assumes that the height of the terminal portion of the $\mathrm{CO}$ profile is related to the number of stars at each galactic longitude in the age range 20-30 $\times 10^{6}$ years per unit of radial velocity. The lower panel shows the fraction of the stars at each galactic longitude in this age range and within $50 \mathrm{~km} \mathrm{~s}^{-1}$ of the highest velocity one from the model. 


\section{CONCLUSIONS}

We have attempted to explain the radial velocity of the terminal portion of the $\mathrm{CO}$ profile relative to that for the $\mathrm{H} \mathrm{I}, 21 \mathrm{~cm}$ line in the region $l=30^{\circ}$ to $60^{\circ}$, $b=0^{\circ}$. On the hypothesis that the $\mathrm{CO}$ kinematics are the same as those for $\mathrm{H}$, velocity differences are interpreted as distance displacements along the line-of-sight using a model for the $\mathrm{H}$ I velocity field. Although one cannot absolutely rule out this hypothesis, it can definitely be said that $\mathrm{CO}$ is not found exclusively in the spiral pattern or any organized structure displaced from the spiral pattern.

Alternatively, because of the association of $\mathrm{CO}$ molecular clouds with regions containing young stars and the fact that infrared sources are found inside them, an explanation involving star dynamics was attempted. In the context of the model used, this attempt succeeded in associating the $\mathrm{CO}$ sources with "stars" of ages (time since initial formation of the dense protostellar cloud in the shock front) less than 30 million years for the region from $l=30^{\circ}$ to $47^{\circ}$. It would be a vast overstatement to say that this agreement offers detailed proof of the correctness of the model. Much work remains to be done in testing how changes in the model parameters affect the predicted star positions and velocities.

It does seem possible to state that if stars are born in spiral arms, and if $\mathrm{CO}$ sources are associated with the process of stellar birth, then their ages must be sufficient to allow them to travel into the space between the spiral arms where they are seen. The 20-30 million year "age" for the CO sources which the model implies greatly exceeds the pre-mainsequence evolution time for stars of spectral type B (Iben 1965) with which the CO sources are associated. Thus the time since the beginning of the collapse of the precluster or preassociation cloud is much less than $20 \times 10^{6}$ years. Iben $(1965)$ finds that the age range $20-30 \times 10^{6}$ years corresponds to the evolutionary lifetime for a star of from 1.5 to $1.25 M_{\odot}$.

We can compare the ages of clusters with which $\mathrm{CO}$ is associated-e.g., NGC 2264, age $\sim 2 \times 10^{6}$ years (Walker 1956); Orion, age $\sim 2 \times 10^{6}$ years (Walker 1969); and $\rho$ Ophiuchi, age less than $10 \times 10^{6}$ years (Carrasco, Strom, and Strom 1973) - against, say, the Pleiades, age $\sim 10^{8}$ years (Sandage 1957), in which no
$\mathrm{CO}$ is found in the central portion. (The $\mathrm{CO}$ data are summarized by Penzias 1975.)

The very close agreement between the $\mathrm{CO}$ terminal velocities and the radial velocity of stars whose ages are less than $30 \times 10^{6}$ years suggests that this is a "cutoff age"- -beyond which a cluster or association is no longer an observable radiator of millimeter wavelength $\mathrm{CO}$ spectral lines. In the region $l<42^{\circ}$, this cutoff age is very sharply defined and a variation of less than 20 percent significantly worsens agreement with the observations. If the cutoff age is so sharply defined in the region $l<42^{\circ}$, why are CO sources seen beyond $l=47^{\circ}$, where the youngest star is $73 \times 10^{6}$ years old? In Figure 11, the dip in the stellar velocities beginning at about $l=43^{\circ}$ can be removed if one starts to consider stars older than $30 \times 10^{6}$ years beginning at $l=43^{\circ}$. This, together with the old stars beyond $l=47^{\circ}$, suggests that either the cutoff age varies as a function of $l$, or the cutoff process is not so steeply time-dependent beyond $l \approx 40^{\circ}$. If the cutoff process is that process by which the cluster drives out the $\mathrm{CO}$, the less steep time-dependence of the process, together with the smaller numbers of young stars, may help explain the decreasing strength of the $\mathrm{CO}$ spectral lines as $l$ increases and the presence of $\mathrm{CO}$ spectral lines beyond $l=47^{\circ}$ where the model finds no young stars.

It is suggested that this $30 \times 10^{6}$ year time corresponds with a time required for the cluster to drive out the $\mathrm{CO}$ which existed in the interstellar medium out of which the cluster formed. Alternatively, it is possible that the main-sequence arrival of the cluster's stars in the mass range $1.25-1.5 M_{\odot}$ extinguishes the source of $\mathrm{CO}$ millimeter line radiation. It would be interesting to observe young clusters whose ages are near $30 \times 10^{6}$ years for the presence of millimeter $\mathrm{CO}$ spectral lines. Finally, it should be pointed out that $30 \times 10^{6}$ years is longer than the entire lifetime of massive $O$ and $B$ stars. If they eject the $\mathrm{C}$ and $\mathrm{O}$ which they produce into the cluster, and if these can form molecules of $\mathrm{CO}$, the preexisting interstellar $\mathrm{CO}$ in the cluster is being enriched by that produced by the massive stars.

It is a pleasure to acknowledge helpful conversations with Tom Barnes, Michel Breger, William Jeffreys, and Paul Vanden Bout. This work was supported in part by NSF grant MPS 72-05070.

\section{REFERENCES}

Burton, W. B., Gordon, M. A., Bania, T. M., and Lockman, F. J. 1975, Ap. J., 202, 30 .

Carrasco, L., Strom, S. E., and Strom, K. E. 1973, Ap. J., 182, 95 .

Contopoulos, G., and Strömgren, B. 1965, Tables of Plane Galactic Orbits (New York: Institute for Space Studies).

Iben, I. 1965, Ap. J., 141, 993.

Penzias, A. 1975, in Physics and Chemistry of the Interstellar Medium, ed. R. Balian (Amsterdam: North-Holland), in press.

Sandage, A. 1957, Ap. J., 125, 435.

Scoville, N. Z., and Solomon, P. M. 1976, Ap. J., in press.

Schmidt, M. 1965, in Galactic Structure, ed. A. Blaauw and M. Schmidt (Chicago: University of Chicago Press).
Shane, W. W., and Bieger-Smith, G. P. 1966, Bull. Astr. Inst. Netherlands, 18, 263.

Shu, F. H., Milione, V., Gebel, W., Yuan, C., Goldsmith, D. W., and Roberts, W. W. 1972, Ap. J., 173, 557.

Shu, F. H., Milione, V., and Roberts, W. W. 1973, Ap.J., 183, 819. Walker, M. F. 1956, Ap. J. Suppl., 2, 365.

1969, Ap. J., $155,447$.

Westerhout, G. 1969, Maryland-Green Bank Galactic 21 Centimeter Line Survey (2d ed.; College Park: University of Maryland).

Yuan, C. 1969a, Ap. J., 158, 871

Y 1969b, Ap. J., 158, 889.

Zuckerman, B., and Palmer, P. 1974, Ann. Rev. of Astr. and Ap., 12, 282.

Frank N. Bash and William L. Peters: Department of Astronomy, University of Texas, Austin, TX

78712 